\title{
Mental Stress from Animal Experiments: a Survey with Korean Researchers
}

\author{
Minji Kang ${ }^{1}$, AhRam Han ${ }^{1}$, Da-eun Kim ${ }^{1}$, Troy Seidle ${ }^{2}$, Kyung-Min Lim ${ }^{1,2}$ and SeungJin Bae' \\ ${ }^{1}$ College of Pharmacy, Ewha Womans University, Seoul, Korea \\ ${ }^{2}$ Research and Toxicology Department, Humane Society International, Toronto, Canada
}

\begin{abstract}
Animal experiments have been widely conducted in the life sciences for more than a century, and have long been a subject of ethical and societal controversy due to the deliberate infliction of harm upon sentient animals. However, the harmful use of animals may also negatively impact the mental health of researchers themselves. We sought to evaluate the anxiety level of researchers engaged in animal use to analyse the mental stress from animal testing. The State Anxiety Scale of the State-Trait Anxiety Inventory (STAI) was used to evaluate how researchers feel when they conduct animal, as opposed to non-animal, based experiments (95 non-animal and 98 animal testing researchers). The Trait Anxiety Scale of STAI was employed to measure proneness to anxiety, namely the base trait of the researchers. Additionally, the information on sex, age, education, income, and total working periods was collected. While the Trait Anxiety scores were comparable (41.5 \pm 10.9 versus $42.9 \pm 10.1, p=0.3682, t$ test), the State Anxiety scores were statistically significantly higher for animal users than non-animal users $(45.1 \pm 10.7$ versus $41.3 \pm 9.4, p=0.011)$. This trend was consistent for both male and female. Notably, younger animal testers ( $\leq 30$ years of age) with less work experience ( $\leq 2$ years) and lower income level $(\leq 27,000$ USD) exhibited higher anxiety scores, whereas these factors did not affect the anxiety level of non-animal users. The present study demonstrated that participation in animal experiments can negatively impact the mental health of researchers.
\end{abstract}

Key words: Animal testing, STAI, State-trait anxiety inventory, Job stress

\section{INTRODUCTION}

Large numbers of animals are sacrificed each year worldwide in biomedical research and safety testing of chemi-

Correspondence to: Kyung-Min Lim, College of Pharmacy, Ewha Womans University, 52 Ewhayeodae-gil, Seodaemun-gu, Seoul 03760, Korea

E-mail:kmlim@ewha.ac.kr

SeungJin Bae, College of Pharmacy, Ewha Womans University, 52

Ewhayeodae-gil, Seodaemun-gu, Seoul 03760, Korea

E-mail: sjbae@ewha.ac.kr

List of abbreviations: STAI, State-Trait Anxiety Inventory; JCQ, Job Content Questionnaire; BDI, Beck Depression Inventory; JSS, the Job Stress Survey.

This is an Open-Access article distributed under the terms of the Creative Commons Attribution Non-Commercial License (http:// creativecommons.org/licenses/by-nc/3.0) which permits unrestricted non-commercial use, distribution, and reproduction in any medium, provided the original work is properly cited. cals and other regulated products. According to Taylor et al. (1), the total number of animals sacrificed for the experiments was estimated to be upwards of 115.3 million worldwide in 2005. Statistical reports in most countries reflect a steady increase in animal use, particularly within the academic sector with the creation and continuous breeding of genetically modified strains of mice and other animals, and this trend has triggered calls for stronger measures to prevent non-vital research and increased investment in a human biology-based research and testing paradigm and associated scientific tools and technologies $(2,3)$. Governmental responses have to date included legislative restrictions on cosmetic animal testing and trade in 35 countries or markets (4), launch of the U.S. interagency 'Tox21' collaboration between the National Institutes of Health, Environmental Protection Agency and Food and Drug Administration (5), and announcements of a national animal reduction strategy in the United Kingdom (6).

Beyond issues on their ethical (7) and scientific (8) jus- 
tifications, animal experiments may also impose a negative impact on the mental health of individuals involved in animal use. Researchers often subject animals to potentially painful and distressing procedures over the course of experiments, and most animals are ultimately sacrificed at the experiment's conclusion. For some researchers, this harmful use of animals goes against their personal moral code, resulting in feelings of internal conflict, guilt and remorse. These conditions may result in chronic job stress as suggested by Rollin (9), who demonstrated that mental stresses can be incurred from breaching of personal ethics and compunction. For example, Reeve et al. (10) reported that substantial proportion of workers in animal shelters (44\% of 335 people) involved in euthanizing animals reported feeling a level of stress comparable to that incurred by "hit by a truck." In this context, it is highly probable that the conduct of animal experiments may negatively impact the mental health of researchers, yet few studies appear to have addressed this issue.

Job stress can be closely linked with individual health conditions and organisational productivity. Workers with job stress drink more alcohol and may experience higher occurrences of hyperlipidemia, sleep disorder and mental depression $(11,12)$. Job stress is also negatively correlated with job satisfaction (13) and can eventually result in avoidance behaviour towards anxiety-provoking situations (14), potentially leading to job instability and productivity loss at the occupational level. Job stresses are broadly investigated across different occupational groups. However, there is no universally accepted definition for job stress (15), and each researcher has developed his/her own definition. Job stress can be illustrated as the psychological distress incurred from the interaction between organisational stressors (e.g., work overload, role conflict, and level of participation) and individual intrinsic stressors (e.g., personality, and family problems) $(16,17)$. To evaluate these multi-factorial stressors, several types of instruments have been employed, such as Karasek's Job Content Questionnaire (JCQ), Beck Depression Inventory (BDI) (12), and the Job Stress Survey (JSS) $(18,19)$. Among these, JCQ was the most frequently used to examine job stress $(20,21)$.

Nonetheless, work environment may vary considerably across different occupational groups and specific stressful situations, such as "disruptive students" for teachers and "work shift" for nurses, can occur (22). As such, the JCQ may not be applicable in all cases. For such outliers, anxiety as one major aspect of mental stress (23) can be measured as a surrogate index for job stress, which is directed towards specific stressful events. The State-Trait Anxiety Inventory (STAI), which measures the anxiety perceived by respondents incurred from specific events, is the representative tool for evaluating anxiety ensued from job stress $(24,25)$. STAI also provides the information about baseline personality (trait anxiety), so the influence of individual stressors can be assessed as well.

Here we examined the effects of laboratory animal experiments on the anxiety level of researchers with STAI in comparison with non-animal-based experiments in an effort to illuminate the job stress and negative impact of animal experiments on the mental health of workers.

\section{MATERIALS AND METHODS}

Study protocol and questionnaire development. The study protocol was reviewed and approved by the Institutional Review Board of Ewha Womans University (IRB no. 123-6). The survey was composed of two parts: sociodemographic questions and State-Trait Anxiety Inventory (STAI). The STAI was used to measure the anxiety of researchers, and has been translated and adapted into more than 40 languages (26). The most recent Korean version of STAI-KYZ, which was developed by Han et al. (27) was employed in this study. The current version of STAIKYZ (including original STAI) is available at http:// www.mindgarden.com/145-state-trait-anxiety-inventoryfor-adults.

The STAI is separated into two-parts for measuring state and trait anxiety. State anxiety is a transitory emotion characterised by physiological arousal $(24,28)$. State anxiety scale evaluates how the respondents feel "right now" with response to external stress, while trait anxiety scale evaluates how the respondents feel "in general," namely the individual's inherent tendency. The state anxiety scale is also useful for measuring anxiety occurring from specific events or imaginary situations. In this study, instructions were provided at the beginning of questionnaire, stating, e.g., "Read each statement and choose the most appropriate answer to indicate how you feel when you conduct animal testing (for animal users) or non-animal testing (for non-animal-users)." Both scales consisted of 20 items. All items were scored on a 4-point Likert-type response scale (from "Not at all" to "Very much so"). Scores ranged from 20 to 80 , with higher scores reflecting a greater level of anxiety.

Participants' trait anxiety scale, ranging from 20 to 80 , was dichotomised as "high anxiety subjects" (score less than 45.8) and "low anxiety subjects" (score equal to or higher than with 45.8) based on the average trait anxiety score of the Korean population in general (27). In previous research, median scores of the American population were used to distinguish the subjects with low and high anxiety $(29,30)$.

Sociodemographic information including gender, age (20 or under vs $21-25$ vs $26-30$ vs $31-35$ vs $36-40$ vs $41-45$ vs $46-50$ vs 50 or older), educational level (high school vs vocational/technical school (2 years), Bachelor's degree, Master's degree, Doctoral degree), annual income (under 20 million KRW, 20-30, 30-40, 40-50, or over 50 million 
KRW), types of experiment mainly involved in (animalvs. non-animal), and total working periods (in months) was collected. In this study, total working years were stratified into three levels: under 2 years, $2 \sim 8$ years and over 8 years, representing early-career, mid-career and late-career stage, respectively, according to A. Cohen's career stage theory (31). Annual income was converted to USD based on an exchange rate of $\$ 1,100 / \$$ (as of September 2016).

Data collection. The target population consisted of scientists working in the bioscience sector at Korean universities, research institutes, as well as cosmetic and pharmaceutical companies. Institutes where animal and nonanimal researchers work together were favoured to reduce the selection bias. Participants involved in animal or nonanimal experiments were included, while those working outside the field of biology were excluded. Each participant was divided into animal or non-animal experiments group by types of experiment they mainly involved in. Organisations consenting to participate were sent questionnaires by email or mail. The first page of the questionnaire was devoted to the information on the study objectives and contents.

The data collection period ran from September through December 2015. The survey was ID-blind; however, mobile phone numbers were collected to provide financial reimbursement (a mobile gift card that amounted to \$4 USD). Mobile numbers were codified to create recipient ID and locked for any further participation. Therefore, researchers could participate in the survey only once. Personal mobile numbers were immediately discarded after reimbursement was provided. All personal information col- lected from the survey was kept confidential.

247 questionnaires were distributed, and 193 researchers were included in the study after 54 incomplete surveys were excluded. 95 non-animal-users and 98 animal users responded in total.

Statistical analyses. Required sample size was estimated based on Cohen's effect size, which can be used at the planning stage to find the required sample size to obtain sufficient power (32,33); 0.8 and 0.05 are conventional values for power and the type 1 error (34). The expected effect size was 0.5 as it indicates a moderate effect. Finally, we followed Cohen's table for estimating sample size in two-tailed $t$-test, which shows that a total sample size of 128 (64 participants per group) is necessary.

Survey response data were summarised using Microsoft Excel $^{\circledR}$ (Microsoft, WA, USA). Incomplete surveys were excluded. State and trait anxiety scores were regarded as continuous variables, while gender (male, female), age (21 30, $\geq 31)$, educational level ( $\leq$ undergrad, $\geq$ postgrad), annual income ( $\leq 27,000$ USD, 27,000 45,000 USD, $\geq 45,000$ USD), career stage ( $\leq 2$ years, $2 \sim 8$ years, $\geq 8$ years) and type of experiment (animal, non-animal) were considered as categorical variables. The age of the respondents were divided as 21 30 and above 30 .

The mean difference between the non-animal user group and animal users was analysed by unpaired $t$-test. Kolmogorov-smirnov test was used to check normality and when the normality cannot be assumed, the Wilcoxon Rank Sum test was used instead of $t$-test. The demographic distribution pattern of participants was analysed by the $\chi^{2}$ test. To analyse the effect of variables with more than 2 levels,

Table 1. Demographic characteristics of the study participants

\begin{tabular}{|c|c|c|c|c|}
\hline \multirow{2}{*}{ Characteristics } & \multicolumn{3}{|c|}{ Number (\%) } & \multirow{2}{*}{$p$-value } \\
\hline & Total $(n=193)$ & Non-animal testing $(\mathrm{n}=95)$ & Animal testing $(\mathrm{n}=98)$ & \\
\hline \multicolumn{5}{|l|}{ Gender } \\
\hline Male & $81(42.0)$ & $30(31.6)$ & $51(52.0)$ & 0.004 \\
\hline Female & $112(58.0)$ & $65(68.4)$ & $47(48.0)$ & \\
\hline \multicolumn{5}{|c|}{ Age group (years) } \\
\hline $21 \sim 30$ & $107(55.4)$ & $61(64.2)$ & $46(46.9)$ & 0.016 \\
\hline$\geq 31$ & $86(44.6)$ & $34(35.8)$ & $52(53.1)$ & \\
\hline \multicolumn{5}{|c|}{ Educational level } \\
\hline$\leq$ Undergrad & $84(43.5)$ & $44(46.3)$ & $40(40.8)$ & 0.441 \\
\hline$\geq$ Postgrad & $109(56.5)$ & $51(53.7)$ & $58(59.2)$ & \\
\hline \multicolumn{5}{|c|}{ Annual income (USD) } \\
\hline$\leq 27,000$ & $106(54.9)$ & $65(68.4)$ & $41(41.8)$ & \\
\hline $27,000 \sim 45,000$ & $46(23.8)$ & $16(16.8)$ & $30(30.6)$ & 0.001 \\
\hline$\geq 45,000$ & $41(21.2)$ & $14(14.7)$ & $27(27.6)$ & \\
\hline \multicolumn{5}{|c|}{ Working period (years) } \\
\hline$\leq 2$ & $74(38.3)$ & $44(46.3)$ & $30(30.6)$ & \\
\hline $2 \sim 8$ & $78(40.4)$ & $37(38.9)$ & $41(41.8)$ & 0.031 \\
\hline$\geq 8$ & $41(21.2)$ & $14(14.7)$ & $27(27.6)$ & \\
\hline
\end{tabular}

$p$ values were estimated based on the $\chi 2$ test. 
one-way ANOVA test was used followed by Bonferroni post-hoc analysis to determine which group was significantly different among groups. Differences were deemed statistically significant at $p<0.05$. All statistical analyses were performed using SAS version 9.4 (SAS Institute Inc, Cary, NC, USA).

\section{RESULTS}

Impact of laboratory animal experiments on the anxiety level of researchers was examined with STAI questionnaire in comparison with non-animal-based experiments. Table 1 shows the demographic characteristics of participants. Females were dominant in the non-animal-user group $(68.4 \%)$, while those involved in animal use were more likely to be male (52.0\%). Age of respondents ranged from 21 to 50 , although the great majority were under 40 (94.3\%). Researchers who were in their earlycareer stage (working experience 2 years or less), midcareer (working experience 2 to 8 years) and late-career stages (8 years or longer) constituted $38.3 \%, 40.4 \%$, and $21.2 \%$ of the sample, respectively.

The mean state anxiety scores are presented in Table 2 , which is further stratified by gender, age group, education level, income level and career stage. The mean anxiety score for all participants was $43.2 \pm 10.2$. While the Trait
Anxiety scores were comparable $(41.5 \pm 10.9$ versus $42.9 \pm$ $10.1, p=0.3682, t$-test), non-animal-users showed anxiety scores of $41.3 \pm 9.4$ while the animal user group had scores of $45.1 \pm 10.7$, which reached statistically significant difference $(p=0.011)$. Regardless of the gender of the researchers, animal users' anxiety score was significantly higher than that of non-animal-users ( $p$-value being 0.006 and 0.043 for males and females, respectively). The anxiety level was highest in the female animal user group, followed by females in the non-animal-user group, male animal users, and male non-animal users (Fig. 1A).

Within the animal user group, state anxiety scores were significantly higher among younger researchers $(\leq 30$ years) with less working experience $(\leq 2$ years) and lower income level ( $\leq 27,000$ USD), whereas significant differences were not observed in other subgroups (Fig. 1B-1D). Ages did not affect the anxiety level for non-animal-users $(p=0.175$, Fig. 1B), but animal users in their twenties scored $47.8 \pm 10.7$, which was significantly higher than for the later-stage scientist group $(42.7 \pm 10.1, p=0.017)$. As a result, anxiety scores of young animal users were significantly higher than for young non-animal users $(p<0.001)$, although this difference became less significant as they grew old.

The mean anxiety scores adjusted for working periods also followed the same pattern (Fig. 1C). Working period

Table 2. Anxiety scores of researchers measured by the state anxiety scores of STAI ${ }^{\dagger}$ (animal testing vs. non-animal)

\begin{tabular}{|c|c|c|c|}
\hline & Non-animal testing $(\mathrm{n}=95)$ & Animal testing $(\mathrm{n}=98)$ & $p$-value \\
\hline All & $41.3 \pm 9.4$ & $45.1 \pm 10.7$ & $\mathbf{0 . 0 1 1}^{\mathrm{a}}$ \\
\hline \multicolumn{4}{|l|}{ Gender } \\
\hline Male & $37.0 \pm 9.2$ & $42.8 \pm 8.7$ & $0.006^{\mathrm{a}}$ \\
\hline Female & $43.3 \pm 8.8$ & $47.6 \pm 12.0$ & $0.043^{\mathrm{a}}$ \\
\hline \multicolumn{4}{|c|}{ Age group (years) } \\
\hline $21 \sim 30$ & $40.4 \pm 8.3$ & $47.8 \pm 10.7$ & $<0.001^{\mathrm{a}}$ \\
\hline$\geq 31$ & $43.1 \pm 10.9$ & $42.7 \pm 10.1$ & $0.857^{\mathrm{a}}$ \\
\hline \multicolumn{4}{|c|}{ Educational level } \\
\hline$\leq$ Undergrad & $40.5 \pm 8.4$ & $45.4 \pm 12.5$ & $0.042^{\mathrm{a}}$ \\
\hline$\geq$ Postgrad & $42.1 \pm 10.2$ & $44.9 \pm 9.3$ & $0.135^{\mathrm{a}}$ \\
\hline \multicolumn{4}{|c|}{ Annual Income (USD) } \\
\hline$\leq 27,000$ & $42.0 \pm 8.5$ & $49.1 \pm 10.4$ & $<0.001^{\mathrm{a}}$ \\
\hline $27,000 \sim 45,000$ & $40.3 \pm 12.0$ & $43.6 \pm 10.3$ & $0.125^{\mathrm{b}}$ \\
\hline$\geq 45,000$ & $39.7 \pm 10.2$ & $40.6 \pm 9.5$ & $0.826^{\mathrm{b}}$ \\
\hline \multicolumn{4}{|c|}{ Working period (years) } \\
\hline$\leq 2$ & $40.9 \pm 9.2$ & $51.2 \pm 8.8$ & $<0.001^{\mathrm{a}}$ \\
\hline $2 \sim 8$ & $42.8 \pm 9.3$ & $41.8 \pm 10.8$ & $0.662^{\mathrm{a}}$ \\
\hline$\geq 8$ & $38.6 \pm 10.0$ & $43.2 \pm 9.8$ & $0.156^{\mathrm{b}}$ \\
\hline
\end{tabular}

Values represent mean \pm SD. ${ }^{\dagger}$ STAI represents State Trait Anxiety Inventory.

${ }^{a}$ Unpaired $t$-test. ${ }^{b}$ Wilcoxon rank sum test. Wilcoxon rank sum test was applied when the normality assumption was failed. 
(A)

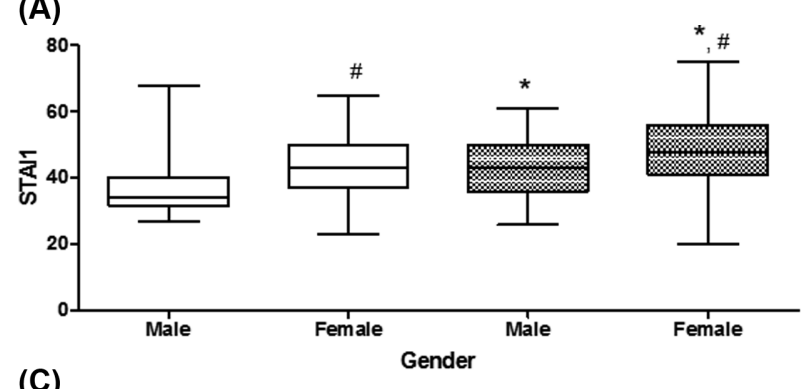

(C)

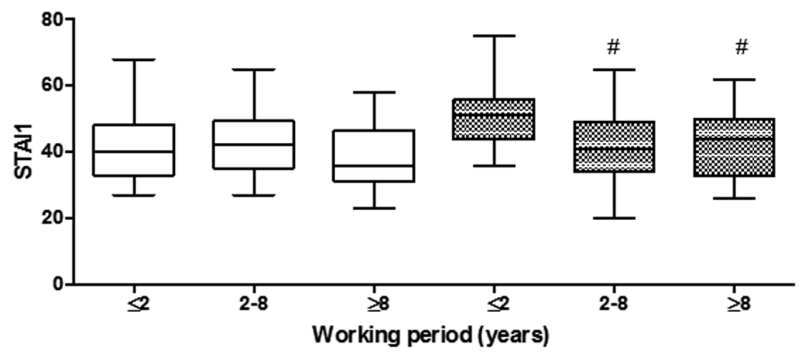

Non-animal Testing
(B)

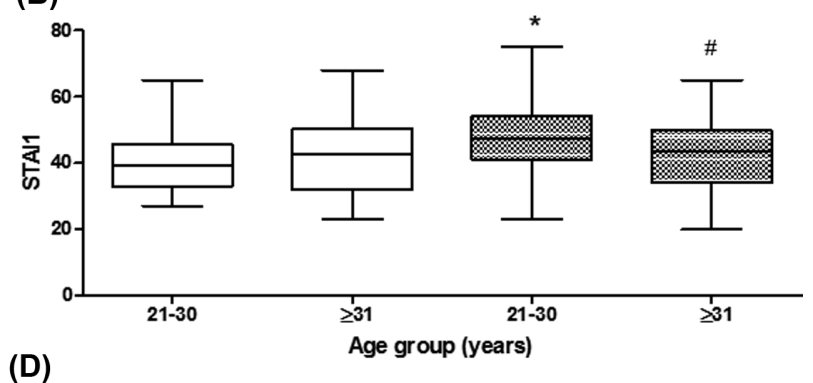

(D)

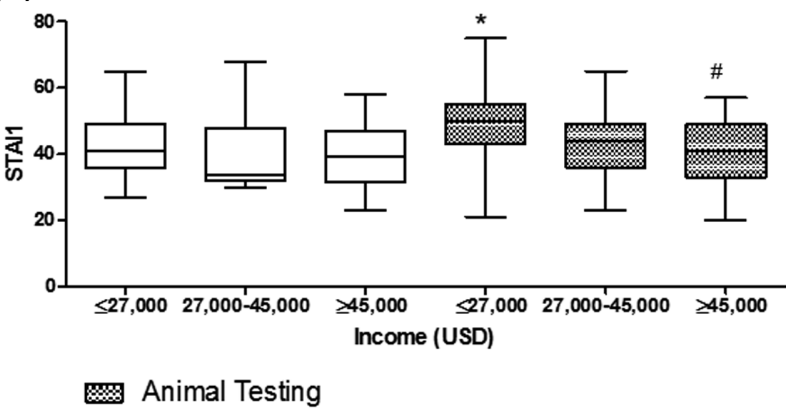

Fig. 1. Comparison of anxiety scores between non-animal testing and animal testing researchers. The state anxiety scores are shown by (A) gender, (B) age group, (C) working experience, and (D) income. Data were presented by the box-whisker plots, with upper or lower whisker extending to the highest or lowest data point with the upper (Q3 + 1.5 (Q3 - Q1)) or lower bound (Q1 - 1.5 $(\mathrm{Q} 3-\mathrm{Q} 1)$ ). *Significantly different between groups (non-animal and animal users) at $p<0.05$. \#Significantly different within a group at $p<0.05$. $t$-test for gender and age, ANOVA for working period and income followed by Bonferroni post-hoc comparisons. STAI1 $=$ State Anxiety Scores of STAI.

had a significant effect on the anxiety level only for animal users ( $p<0.001$, ANOVA analysis). Bonferroni posthoc analysis was applied and the difference was noted in the animal user group with less than 2 years of work experience as compared to those with more experience. Similar to the effects of age on anxiety, the difference between non-animal and animal use was greatest for those in early career stages, and the difference became less significant as experience increased.

Income level also influenced the anxiety level of animal users (Fig. 1D). Only within the lowest income group was there a significant difference between animal- and nonanimal uses. Since age, working period and income were highly correlated factors, it can be concluded that those who just started their career were affected most by anxiety incurred from animal use, and this heightened anxiety seems to dissipate as they reach their mid-career stage.

Trait anxiety significantly affects the state anxiety level and there is a significant correlation between them, which means that individual's anxiety reaction can depend in part on the level of his/her trait (28). To adjust the effect of trait anxiety on the level of state anxiety, respondents were split into two groups: those with low and high anxiety traits. Low/high anxiety traits were divided according to the mean trait anxiety score of Korean population (Mean trait anxiety score $=45.8$ ) (27) Differences between nonanimal and animal users achieved statistical significance among low-anxiety subjects $(37.3 \pm 7.3$ versus $41.5 \pm 9.9$, $p=0.0075$ ), while in the highly anxious group, only limited extent of difference was noted $(47.9 \pm 8.6$ versus $52.0 \pm 8.6, p=0.0522$ ).

\section{DISCUSSION}

Our study demonstrated that participation in animal experiments might have significant, anxiety-provoking situation especially for the workers in their early stages. Gender differences were pronounced, with female researchers exhibiting higher anxiety levels than their male counterparts in both animal- and non-animal-user contexts. This difference is in line with the previous study demonstrating that the trait anxiety is higher in woman then in men $(14,35)$. Gender difference may not only affect trait anxiety, but result in the different emotional and cognitive reactions towards animal experiments. According to Gallop and Beckstead, female students were more concerned about animal welfare and more sympathetic toward animals' pain and suffering (36). For example, more female students agreed with the statement "I am very concerned about pain and suffering in animals" than male ( $82 \%$ versus $65.9 \%$ ). In addition, fewer female students were positive towards animal use for testing or health research $(59.2 \%$ versus $80.9 \%$ for male). This matches well with our results and strongly supports that female workers may 
experience higher levels of anxiety and stress as a result of involvement in the harmful use of animals (36).

Interestingly, this study also revealed that stress levels of researchers incurred from animal use may be different depending on their socioeconomic status. Younger or less experienced researchers reported higher degrees of anxiety from animal experiments. Researchers in their earlycareer stage were more affected by animal experiments than those in their mid- and late-career stages. This phenomenon can be explained by adaptation and desensitisation. Technical skills and competence can be improved and psychological burden rising from unskilled animal use can be resolved $(31,37)$. For those who were able to adapt and become desensitised, animal experiments might have become part of their daily work routine. Conversely, less experienced researchers who did not adapt may be driven to resign their position and seek out another that is more in line with their personal ethical values.

Lastly, we demonstrated that conducting animal experiments may be a stress-provoking situation regardless of individuals' proneness to anxiety. Since a strong positive correlation between state anxiety and trait anxiety was shown ( $\mathrm{r}=0.644$ for non-animal, $\mathrm{r}=0.699$ for animal testing, $p<0.01$, Pearson's Correlation, data not shown), the contribution of researchers' baseline trait cannot be neglected. When we divided respondents into two groups, participation in animal experiments can be a reason for greater anxiety even within groups with similar trait anxiety. Moreover, animal experimentation had significant impacts on low anxiety subjects, suggesting that even the subjects with relatively stable mental status were also affected. However, it shall be interpreted with caution since the subject number became smaller after stratification.

To our best knowledge, cut-off scores determining the clinically meaningful state anxiety level have not as yet been validated. Previously, clinically meaningful state anxiety level was determined as one standard deviation above the mean (38). Among the Korean population the mean state anxiety score was 44.27 and the standard deviation, 9.85 (27). As such, scores greater than 54.12 could be classified as having clinically meaningful anxiety level. $18.4 \%$ of animal experiment workers scored above this cut-off value as compared to $8.4 \%$ of non-animal, suggesting that those mainly involved in animal experiments may be significantly more prone to experiencing anxiety disorders. This phenomenon warrants further examination.

This study has several limitations. In this study, anxiety was used as an indicator of overall stress. However, volume of such work undertaken, time spent on animal related work, severity of procedures should be considered to understand overall mental stress from animal experiment. Secondly, this study includes a relatively small sample population, which cannot accurately represent entire population. Further research with larger, and representa- tive sample is needed to confirm our findings. Response bias may have occurred because of the nature of the assessment. Poulton indicated the participants may avoid selecting the extreme categories at each end of the rating scale, reducing the capacity to discriminate between the stimuli (39). It appears particularly and evidently true with the case of the rating scale such as STAI (38). Finally, this study cannot provide clear reasons to explain the statistical differences between groups. As such, further investigation using refined study designs and larger sample numbers is needed.

Notwithstanding with these limitations, to our best knowledge, this study is the first attempt to evaluate the mental stress of animal experiments on researchers themselves. There have been many interventions against animal experiments from different stakeholders' perspectives, but most have focused on the scientific progress, ethics, animal welfare or marketing strategy (40) while the mental health of researchers themselves has been neglected. The strength of our study lies in providing an insight into the negative effects of animal experimentation on the mental health of researchers, which will be inspiring for further research on the potential adverse impacts of animal experimentation on scientific innovation, industrial competitiveness, and society as a whole.

\section{ACKNOWLEDGMENTS}

This research was supported by grant, 17182MFDS489 from the Ministry of Food \& Drug Safety of Korea.

\section{CONFLICT OF INTEREST}

None to declare.

Received November 22, 2017; Revised December 24, 2017; Accepted January 2, 2018

\section{REFERENCES}

1. Taylor, K., Gordon, N., Langley, G. and Higgins, W. (2008) Estimates for worldwide laboratory animal use in 2005.

2. Russell, W.M.S., Burch, R.L. and Hume, C.W. (1959) The Principles of Humane Experimental Technique, Methuen.

3. Seidle, T. (2013) Humane Society International's global campaign to end animal testing. Altern. Lab. Anim., 41, 453459.

4. EC (2013) Regulation (EC) No 1223/2009 of the European Parliament and of the Council of 30 November 2009 on cosmetic products (Commission E, Ed.). European Union law.

5. Collins, F.S., Gray, G.M. and Bucher, J.R. (2008) Toxicology. Transforming environmental health protection. Science, 319, 906-907.

6. Törnqvist, E., Annas, A., Granath, B., Jalkesten, E., Cotgreave, I. and Öberg, M. (2014) Strategic focus on 3R 
principles reveals major reductions in the use of animals in pharmaceutical toxicity testing. PLOS ONE, 9, e101638.

7. Pound, P., Ebrahim, S., Sandercock, P., Bracken, M.B. and Roberts, I. (2004) Where is the evidence that animal research benefits humans? BMJ, 328, 514-517.

8. Olson, H., Betton, G., Robinson, D., Thomas, K., Monro, A., Kolaja, G., Lilly, P., Sanders, J., Sipes, G., Bracken, W., Dorato, M., Van Deun, K., Smith, P., Berger, B. and Heller, A. (2000) Concordance of the toxicity of pharmaceuticals in humans and in animals. Regul. Toxicol. Pharmacol., 32, 5667.

9. Rollin, B.E. (1987) Euthanasia and moral stress. Loss, Grief \& Care, 1, 115-126.

10. Reeve, C.L., Rogelberg, S.G., Spitzmüller, C. and DiGiacomo, N. (2005) The caring-killing paradox: euthanasia?related strain among animal-shelter workers. J. Appl. Soc. Psychol., 35, 119-143.

11. Nomura, K., Nakao, M., Tsurugano, S., Takeuchi, T., Inoue, M., Shinozaki, Y. and Yano, E. (2010) Job stress and healthy behavior among male Japanese office workers. Am. J. Ind. Med., 53, 1128-1134.

12. Tsai, S.-Y. (2012) A study of the health-related quality of life and work-related stress of white-collar migrant workers. Int. J. Environ. Res. Public Health, 9, 3740-3754.

13. Johnson, S., Cooper, C., Cartwright, S., Donald, I., Taylor, P. and Millet, C. (2005) The experience of work-related stress across occupations. J. Manage. Psychol., 20, 178-187.

14. Muschalla, B., Linden, M. and Olbrich, D. (2010) The relationship between job-anxiety and trait-anxiety--a differential diagnostic investigation with the Job-Anxiety-Scale and the State-Trait-Anxiety-Inventory. J. Anxiety Disord., 24, 366-371.

15. Parker, D.F. and DeCotiis, T.A. (1983) Organizational determinants of job stress. Organ. Behav. Hum. Perform., 32, 160-177.

16. Schuler, R.S. (1980) Definition and conceptualization of stress in organizations. Organ. Behav. Hum. Perform., 25, 184-215.

17. Finney, C., Stergiopoulos, E., Hensel, J., Bonato, S. and Dewa, C.S. (2013) Organizational stressors associated with job stress and burnout in correctional officers: a systematic review. BMC Public Health, 13, 1.

18. Silva, A.A., Souza, J.M., Borges, F.N. and Fischer, F.M. (2010) Health-related quality of life and working conditions among nursing providers. Rev. Saude Publica, 44, 718-725.

19. Bragard, I., Dupuis, G., Razavi, D., Reynaert, C. and Etienne, A.-M. (2012) Quality of work life in doctors working with cancer patients. Occup. Med., 62, 34-40.

20. Fernandes, M.H. and Rocha, V.M. (2009) Impact of the psychosocial aspects of work on the quality of life of teachers. Rev. Bras. Psiquiatr., 31, 15-20.

21. Liang, Y.-W., Hsieh, Y., Lin, Y.-H. and Chen, W.-Y. (2014) The impact of job stressors on health-related quality of life of nursing assistants in long-term care settings. Geriatr. Nurs., 35, 114-119.

22. Tennant, C. (2001) Work-related stress and depressive disorders. J. Psychosom. Res., 51, 697-704.

23. Margolis, B.L., Kroes, W.H. and Quinn, R.P. (1974) Job stress: an unlisted occupational hazard. J. Occup. Environ. Med., 16, 659-661.

24. Endler, N.S. and Kocovski, N.L. (2001) State and trait anxiety revisited. J. Anxiety Disord., 15, 231-245.

25. Julian, L.J. (2011) Measures of anxiety: State-Trait Anxiety Inventory (STAI), Beck Anxiety Inventory (BAI), and Hospital Anxiety and Depression Scale-Anxiety (HADS-A). Arthritis Care Res (Hoboken), 63, S467-S472.

26. Spielberger, C.D. and Sydeman, S.J. (1994) State-trait anxiety inventory and state-trait anger expression inventory in The Use of Psychological Testing for Treatment Planning and Outcome Assessment (Maruish, M.E. Ed.). Lawrence Erlbaum Associates, Hillsdale, New Jersey, pp. 292-321.

27. Hahn, D.-W., Lee, C.-H. and Chon, K.-K. (1996) Korean adaptation of Spielberger's STAI (K-STAI). Korean $J$. Health Psychol., 1, 1-14.

28. Spielberger, C.D., Gorsuch, R.L. and Lushen, R.E. (1970) Manual for the State-Trait Anxiety Inventory, Consulting Psychologists Press, Inc., Palo Alto, CA.

29. Weinstein, A.M. (1995) Visual ERPs evidence for enhanced processing of threatening information in anxious university students. Biol. Psychiatry, 37, 847-858.

30. Addolorato, G., Capristo, E., Stefanini, G. and Gasbarrini, G. (1997) Inflammatory bowel disease: a study of the association between anxiety and depression, physical morbidity, and nutritional status. Scand. J. Gastroenterol., 32, 10131021.

31. Cohen, A. (1991) Career stage as a moderator of the relationships between organizational commitment and its outcomes: a meta-analysis. J. Occup. Organ. Psychol., 64, 253-268.

32. Cohen, J. (1992) Statistical power analysis. Curr. Dir. Psychol. Sci., 1, 98-101.

33. Sullivan, G.M. and Feinn, R. (2012) Using effect size-or why the $\mathrm{P}$ value is not enough. J. Grad. Med. Educ., 4, 279-282.

34. Noordzij, M., Tripepi, G., Dekker, F.W., Zoccali, C., Tanck, M.W. and Jager, K.J. (2010) Sample size calculations: basic principles and common pitfalls. Nephrol. Dial. Transplant., 25, 1388-1393

35. De Visser, L., Van der Knaap, L., Van de Loo, A., Van der Weerd, C., Ohl, F. and Van Den Bos, R. (2010) Trait anxiety affects decision-making differently in healthy men and women: towards gender-specific endophenotypes of anxiety. Neuropsychologia, 48, 1598-1606.

36. Gallop, G.G. and Beckstead, J.W. (1988) Attitudes toward animal research. Am. Psychol., 43, 474.

37. Reeve, C.L., Spitzmuller, C., Rogelberg, S.G., Walker, A., Schultz, L. and Clark, O. (2004) Employee reactions and adjustment to euthanasia-related work: Identifying turningpoint events through retrospective narratives. J. Appl. Anim. Welf. Sci., 7, 1-25.

38. Millar, K., Jelicic, M., Bonke, B. and Asbury, A. (1995) Assessment of preoperative anxiety: comparison of measures in patients awaiting surgery for breast cancer. $\mathrm{Br} . J$. Anaesth., 74, 180-183.

39. Poulton, E. (1982) Biases in quantitative judgements. Appl. Ergon., 13, 31-42.

40. Bottini, A.A. and Hartung, T. (2009) Food for thought... on the economics of animal testing. ALTEX, 26, 3-16. 